\title{
Domination of Cayley Digraph and its Complement
}

\author{
R. Rajeswari, R. Udhayashree, M. Nirmala
}

\begin{abstract}
A Cayley graph constructed out of a group $\Gamma$ and its generating set $A$ is denoted by $C a y(\Gamma, A)$. The digraph with the same node set as the original digraph is said to be a complement digraph if it has an edge from $x$ to $y$ exactly when the original digraph does not have an edge from $x$ to $y$. A subset $D$ of $V$ is called a dominating set if each vertex in $V$ - $D$ is adjacent to at least one vertex in $\boldsymbol{D}$. The minimum cardinality of a dominating set is called Domination number which is denoted by $\gamma$. The domination number of Cayley digraphs and Complement of Cayley digraphs of groups are investigated in this paper. Also, the graph relationship involving domination parameters in a graph and its complement are studied.
\end{abstract}

Keywords: Cayley digraph; Complement of a graph; Domination number; Alternating group.

\section{INTRODUCTION}

The vertex transitivity property of Cayley graph act an important role in interconnection networks, optimization problems etc., in particular, routing problems in parallel computer [4][5][6][9].

The concepts of domination easily generalize to digraphs. Digraphs are, in a sense, a more natural setting for domination since domination is, intuitively, a non-symmetric idea. However, domination in digraphs has not been studied nearly as extensively as domination in graphs.

Domination of graphs[8] are applied to several fields. In facility location problems, we try to minimize the distance travelled by one person in order to acquire to the closest facility with the fixed number of facilities provided. A similar situation occurs when we try to minimize the number of essential facilities in order to service everyone with the fixed maximum distance to a facility. The concepts of domination in graphs is also applied in monitoring electrical networks or communication networks, in land surveying and in situations involving finding sets of representatives.

\section{LITERATURE SURVEY}

Domination was introduced for digraphs by Y.Fu in 1968. Domination of digraphs and Independent perfect domination in digraphs were studied by Changwoo Lee in 1998 and 2001[1]. Condition for existence of efficient dominating sets and Perfect domination in Cayley graphs were studied by Italo J. Dejtera and Oriol Serrab[2]. Inverse domination number of a graph was discussed by G. S. Domke, L. R. Markus and J. E. Dunbar [3].

T.Tamizh Chelvam, et al. analysed domination in some classes of circulant graphs and characterized all 2 - excellent graphs [8].

\section{MAIN RESULTS}

Motivated by the literatures on dimination of various digraphs and their influence in various feilds, in this study non abelian group $\mathrm{A}_{4}$, an alternating group on four elements set say, $\{1,2,3,4\}$ is considered and domination of Cay $(G, X)$ and its complement Cay $\overline{\left(G_{0} X\right)}$ were analysed.

Construct Cay $(G, X)$ for $G=A_{4}$ with $X \subseteq G$. In Cay $\left(\mathrm{A}_{4}, \mathrm{X}\right)$ either $|\mathrm{X}|=5$ or $|\mathrm{X}|=3$. In both the situations one of Cay $(\mathrm{G}, \mathrm{X})$ and Cay $\overline{(G, X)}$ had an efficient dominating set . Therefore, $\mathrm{A}_{4}$ is one among the smallest groups such that domination number of its cayley digraph differs from the domination number of its complement digraph.

Theorem 3.1: Let $A_{4}$, an alternating group on four elements set say, $\{1,2,3,4\}$ and $X=\{(123)$, (12)(34), (243) $\}$. Then $\gamma\left(\right.$ Cay $\left.\left(\mathrm{A}_{4}, \mathrm{X}\right)\right)=3$ and $\gamma\left(\operatorname{Cay}\left(\overline{\left(A_{4}, X\right)}\right)=2\right.$.

\section{Proof:}

Let us denote Cay $\left(\mathrm{A}_{4}, \mathrm{X}\right)$ and Cay $\overline{\left(A_{4}, \bar{X}\right)}$ by $\mathrm{C}_{\mathrm{X}}$ and $\overline{C_{X}}$ respectively. The digraph $\mathrm{C}_{\mathrm{X}}$ is shown in Figure 3.1.

First, we prove $\gamma\left(C_{X}\right)=3$.

Let $S=\{$ id, (134), (143) $\}$.

Then $\mathrm{N}_{\mathbf{C X}}[\mathrm{id}]=\{\mathrm{id},(123),(12)(34),(243)\}$,

$\mathrm{N}_{\mathbf{C X}}[(134)]=\{(134),(234),(142),(124)\}$,

$\mathrm{N}_{\mathbf{C X}}[(143)]=\{(143),(14)(23),(132),(13)(24)\}$.

Hence $\mathrm{N}_{\mathrm{CX}}[\mathrm{S}]=\mathrm{A}_{4}=\mathrm{V}\left(\mathrm{C}_{\mathrm{X}}\right)$, Therefore, $\mathrm{S}$ is a dominating set. Since $\mathrm{V}\left(\mathrm{C}_{\mathrm{X}}\right)=12$, exactly three arcs starts from every vertex of $\mathrm{C}_{\mathrm{X}}$, therefore, the set $\mathrm{S}$ is a minimum dominating set. Hence $\gamma\left(C_{X}\right)=3$. Also $N_{C X}$ [id], $N_{C X}[(143)]$ and $\mathrm{N}_{\mathbf{C X}}[(134)]$ have no common elements. So, $\mathrm{S}$ is an efficient dominating set. Chennai, India

R. Udhayashree, Sathyabama Institute of Science and Technology, Chennai, India

M. Nirmala, Sathyabama Institute of Science and Technology, Chennai, India 


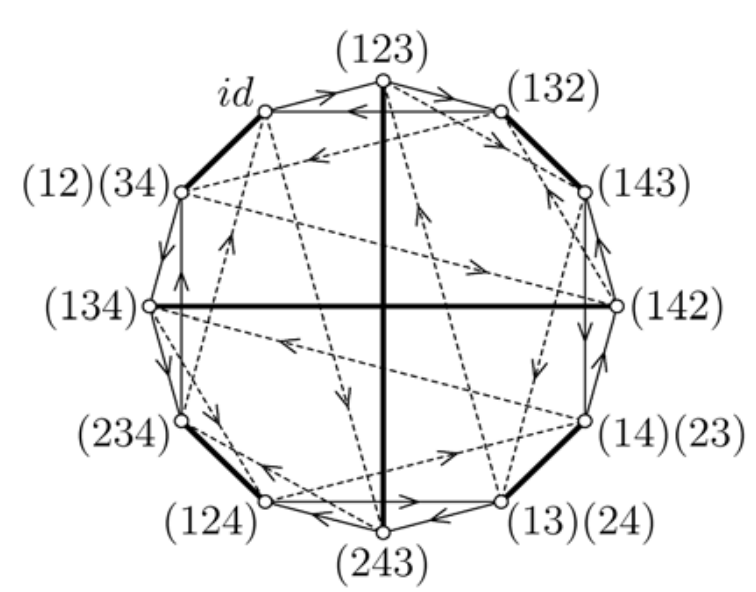

3.1 Cayley digraph $\operatorname{Cay}\left(A_{4}, X\right)$

Now consider $\overline{C_{X}}$, complement graph of $\mathrm{C}_{\mathrm{X}}$, which is shown in 3.2 .

$N_{\bar{C}_{x}}[(\mathrm{id})]=\{(132),(143),(142),(14)(23),(13)(24),(124)$, (234), (134)\},

$N_{\bar{C}_{\mathcal{X}}}[(123)]=\{\mathrm{id},(142),(14)(23),(13)(24),(124),(234)$, (134), (12)(34)\},

$N_{\bar{C}_{\bar{X}}}[(132)]=\{(123),(142),(14)(23),(13)(24),(243),(124)$, (234), (134)\}

$N_{\bar{C}_{\mathcal{X}}}[(143)]=\{\mathrm{id},(123),(13)(24),(243),(124),(234)$, (134), (12)(34)\}

$N_{\bar{C}_{\mathbb{*}}}[(142)]=\{\mathrm{id},(123),(14)(23),(13)(24),(243),(124)$, (234), (12)(34)\}

$N_{\overline{C^{*}}}[(14)(23)]=\{\mathrm{id},(123),(132),(143),(243),(124)$, (234), (12)(34)\}

$N_{\overline{C_{X}}}[(13)(24)]=\{\mathrm{id},(132),(143),(142),(124),(234)$, (134), (12)(34)\}

$N_{\overline{C_{X}}}[(243)]=\{\mathrm{id},(132),(143),(142),(14)(23),(13)(24)$, (134), (12)(34)\}

$N_{\bar{C}_{\mathbb{X}}}[(124)]=\{\mathrm{id},(123),(132),(143),(142),(243),(134)$, (12)(34)\}

$N_{\bar{C}_{\bar{X}}}[(234)]=\{(123),(132),(143),(142),(14)(23),(13)(24)$, (243), (134)\}

$N_{\bar{C}_{\mathcal{X}}}[(134)]=\{\mathrm{id},(123),(132),(143),(14)(23),(13)(24)$, (234), (12)(34)\}

$N_{\bar{C}_{\mathcal{X}}}[(12)(34)]=\{(123),(132),(143),(14)(23),(13)(24)$, (243), (124), (234)\}

The union of neighbourhood of any two non-adjacent vertices of $\overline{C X}$ gives

$\mathrm{V}(\overline{C X})$. Hence $\gamma\left(\right.$ Cay $\left.\overline{\left(A_{4}, X\right)}\right)=2$. Also, the corresponding neighbourhood sets are

not disjoint. Therefore, it is not efficient dominating set.

Hence $\gamma\left(\operatorname{Cay}\left(\mathrm{A}_{4}, \mathrm{X}\right)\right) \neq \gamma\left(\operatorname{Cay} \overline{\left(A_{4}, X\right)}\right)$.

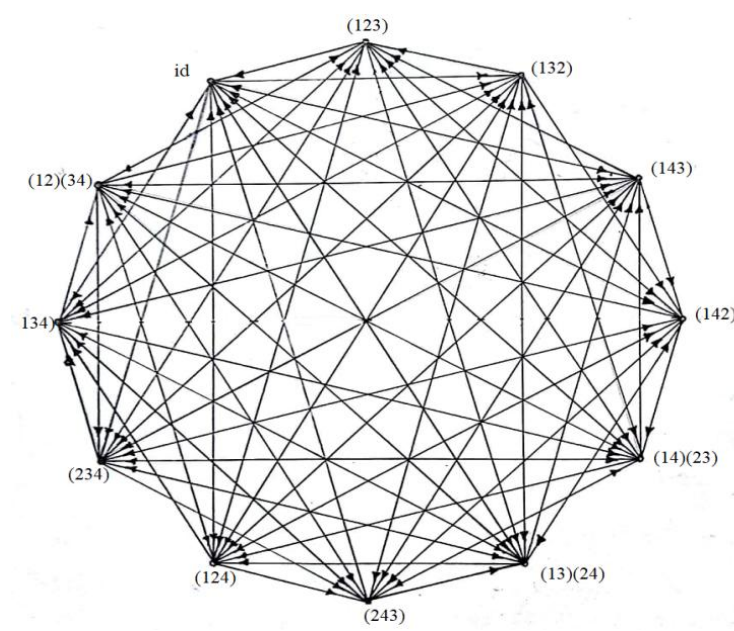

\subsection{Complement of Cayley digraph $\operatorname{Cay}\left(\mathrm{A}_{4}, \mathrm{X}\right)$}

Theorem 3.2: Let $\mathrm{A}_{4}$, an alternating group on four elements set $\{1,2,3,4\}$ and let $Y=(142),\{(14)(23),(13)(24),(134)\}$ then $\gamma\left(\right.$ Cay $\left.\mathrm{A}_{4}, \mathrm{Y}\right)=4$ and $\gamma\left(\mathrm{Cay} \overline{\left(A_{4}, Y\right)}\right)=2$.

\section{Proof:}

Let us denote $\mathrm{C}_{\mathrm{Y}}$ as $\operatorname{Cay}\left(\mathrm{A}_{4}, \mathrm{Y}\right) \& \overline{C_{Y}}$ as $\operatorname{Cay} \overline{\left(A_{4}, Y\right)}$ is shown in Figure 3.3 and 3.4. We examine that $\mathrm{Y}=(\mathrm{X} \cup\{\mathrm{id}\})$ where $X=\{(123),(12)(34),(243)\} \& f=(13)(24)$ clearly $\left(N_{C X}[s]\right) f$ $=\mathrm{N}_{\mathrm{CY}}[\mathrm{s}]$ for every $\mathrm{S} \subseteq \mathrm{A}_{4}$. $\mathrm{S}$ is a total dominating set in $\mathrm{C}_{\mathrm{X}}$ if and only if it is a dominating set in $\mathrm{C}_{\mathrm{Y}}$. Hence $\gamma\left(\mathrm{C}_{\mathrm{Y}}\right)=4$.

$\mathrm{N}_{\mathrm{CY}}[(\mathrm{id})]=\{(142),(14)(23),(13)(24),(134)\}$

$\mathrm{N}_{\mathrm{CY}}[(123)]=\{(142),(124),(234),(134)\}$,

$\mathrm{N}_{\mathrm{CY}}[(132)]=\{(14)(23),(13)(24),(124),(234)\}$

$\mathrm{N}_{\mathrm{CY}}[(143)]=\{\mathrm{id},(124),(234),(12)(34)\}$

$\mathrm{N}_{\mathrm{CY}}[(142)]=\{(123),(243),(124),(234)\}$

$\mathrm{N}_{\mathrm{CY}}[(14)(23)]=\{\mathrm{id},(123),(243),(12)(34)\}$

$\mathrm{N}_{\mathrm{CY}}[(13)(24)]=\{\mathrm{id},(142),(134),(12)(34)\}$

$\mathrm{N}_{\mathrm{CY}}[(243)]=\{(132),(143),(142),(134)\}$

$\mathrm{N}_{\mathrm{CY}}[(124)]=\{\mathrm{id},(132),(143),(12)(34)\}$

$\mathrm{N}_{\text {CY }}[(234)]=\{(132),(143),(14)(23),(13)(24)\}$

$\mathrm{N}_{\mathrm{CY}}[(134)]=\{(123),(132),(143),(234)\}$

$\mathrm{N}_{\mathrm{CY}}[(12)(34)]=\{(123),(14)(23),(13)(24),(243)\}$

$$
\text { So } \mathrm{N}_{\mathrm{CY}}[\mathrm{S}]=\mathrm{A}_{4}=\mathrm{V}\left(\mathrm{C}_{\mathrm{Y}}\right) \text {. Since } \mathrm{V}\left(\mathrm{C}_{\mathrm{Y}}\right)=12 \text { and }
$$
exactly four arcs starts from every vertex of $\mathrm{C}_{\mathrm{Y}}$, the set $\mathrm{S}$ is a minimum dominating set. Hence $\gamma\left(\mathrm{C}_{\mathrm{Y}}\right)=4$. 


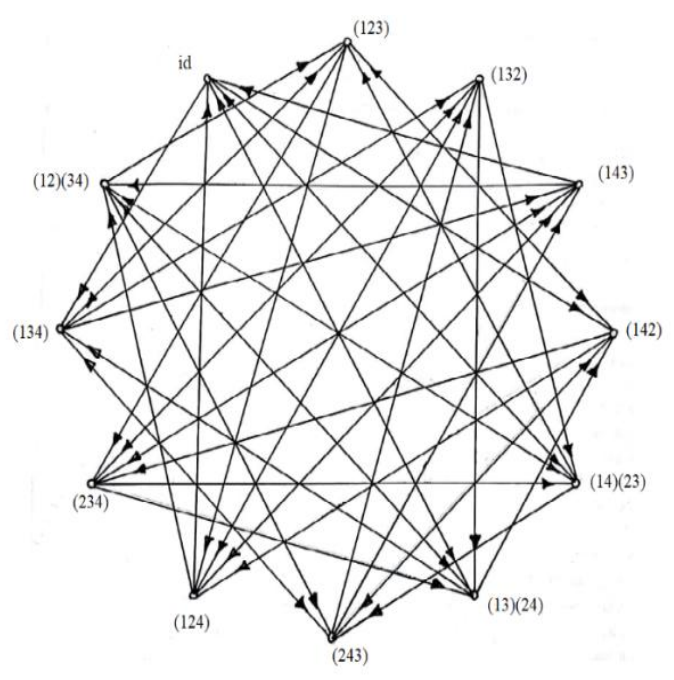

\subsection{Cayley Digraph Cay $\left(\mathrm{A}_{4}, \mathrm{Y}\right)$}

Now consider $\overline{C_{Y}}$, complement graph of $\mathrm{C}_{\mathrm{Y}}$, which is shown in fig 3.4

$N_{\overparen{ }}[(\mathrm{id})]=\{\mathrm{id},(123),(132),(143),(243),(124),(234)$, (12)(34)\}

$N_{\text {CF }}[(123)]=\{\mathrm{id},(123),(132),(143),(14)(23),(13)(24)$, (243), (12)(34)\}

$N_{C Z}[(132)]=\{\mathrm{id},(123),(132),(143),(142),(243),(134)$, $(12)(34)\}$

$N_{C Y}[(143)]=\{(123),(132),(143),(142),(14)(23),(13)(24)$, (243), (134)\}

$N_{\text {CY }}[(142)]=\{\mathrm{id},(132),(143),(142),(14)(23),(13)(24)$, (134), (12)(34)\}

$N_{C Y}[(14)(23)]=\{(132),(143),(142),(14)(23),(13)(24)$, (124), (234), (134) $\}$

$N_{C Y}[(13)(24)]=\{(123),(132),(143),(14)(23),(13)(24)$, (243), (124), (234)\}

$N_{C Y}[(243)]=\{\mathrm{id},(123),(14)(23),(13)(24),(243),(124)$, (234), (12)(34)\}

$N_{C Z}[(124)]=\{(123),(142),(14)(23),(13)(24),(243),(124)$, (234), (134)\}

$N_{C Y}[(234)]=\{\mathrm{id},(123),(142),(243),(124),(234),(134)$, (12)(34)\}

$N_{C Y}[(134)]=\{\mathrm{id},(142),(14)(23),(13)(24),(243),(124)$, (134), (12)(34)\}

$N_{C Y}[(12)(34)]=\{\mathrm{id},(132),(143),(142),(124),(234),(134)$, $(12)(34)\}$

But every vertex of Cay $\overline{\left(A_{4}, Y\right)}$ has seven neighborhoods. So any two vertex covers all the vertices of Cay $\overline{\left(A_{4}, Y\right)}$.

Therefore, $\operatorname{Cay} \overline{\left(A_{4}, Y\right)}=2$.

Corollary 4.3: If $G$ is a Cayley digraph $\operatorname{Cay}(G, S)$ With $|S|=2$ or 3 , then Domination of complement of $\operatorname{Cay}(G, S)$ is always 2 .

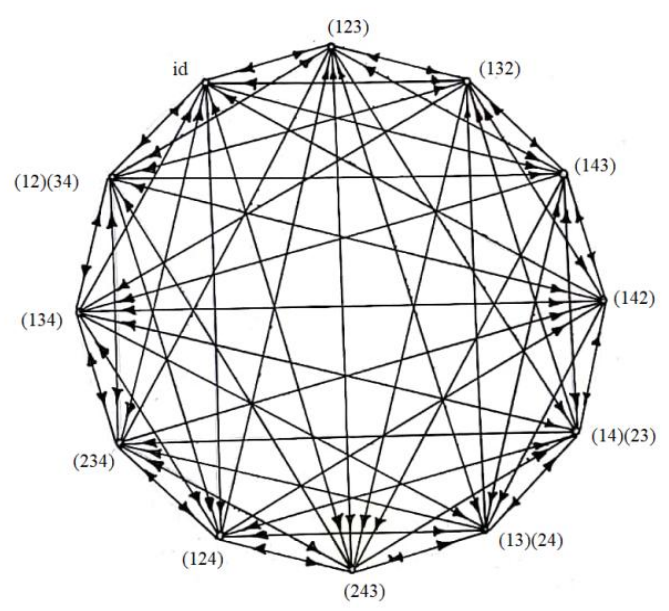

3.4 Complement of Cayley Digraph Cay(A4, Y)

\section{RESULTS AND DISCUSSION}

Let $\mathrm{v}$ be an arbitrary vertex in $\mathrm{G}$ , then the closed neighborhood of $\mathrm{v}$, i.e., $\mathrm{NG}[\mathrm{v}]$ is a dominating set of $\mathrm{G}^{-}$. In particular, Cayley digraph is a vertex transitive, regular digraph, we have $\gamma(\mathrm{G}) \leq \delta(\mathrm{G})+1$. Furthermore, If we consider a set formed by taking a vertex from each color class of any arbitrary $\quad \chi(\mathrm{G})$-coloring of $\mathrm{G}$ then it is also a dominating set of $\mathrm{G}^{-}$, Hence $\gamma(\mathrm{G}) \leq \chi(\mathrm{G})$. The above results leads to the following observation.

Observation 4.1: Let $\mathrm{G}$ be a graph. Then

(a) $\gamma(\mathrm{G} \overline{)} \leq \delta(\mathrm{G})+1$.

(b) $\gamma(\mathrm{G} \overline{)} \leq \chi(\mathrm{G})$.

By Observation (a), $\gamma(\mathrm{G}) \leq \Delta(\mathrm{G})+1$. But by Brook's Coloring Theorem, $\chi(\mathrm{G}) \leq \Delta(\mathrm{G})+1$ with equality if and only if $\mathrm{G}$ is Cayley digraph $\operatorname{Cay}(\mathrm{G}, \mathrm{S})$ with $|\mathrm{S}|=\mathrm{n}-1$ and $|\mathrm{V}(\mathrm{G})|=\mathrm{n}$. Noting that the domination number of the complement of $\operatorname{Cay}(\mathrm{A} 4, \mathrm{~S})$ with $|\mathrm{S}|=2$ or 3 , is equal to 2 , then $\gamma(\mathrm{G}) \leq \Delta(\mathrm{G})$ +1 .

Observation 4.1: Let $\mathrm{G}$ be a graph. Then

(a) $\gamma(\mathrm{G} \overline{)} \leq \delta(\mathrm{G})+1$.

(b) $\gamma(\mathrm{G} \overline{)} \leq \chi(\mathrm{G})$.

By Observation (a), $\gamma(\mathrm{G}) \leq \Delta(\mathrm{G})+1$. But by Brook's Coloring Theorem, $\chi(\mathrm{G}) \leq \Delta(\mathrm{G})+1$ with equality if and only if $G$ is Cayley digraph Cay $(G, S)$ with $|S|=n-1$ and $|V(G)|=n$. Noting that the domination number of the complement of Cay(A4, S) with $|\mathrm{S}|=2$ or 3 , is equal to 2 , then $\gamma(\mathrm{G}) \leq \Delta(\mathrm{G})$ +1 .

Observation 4.2: If $\mathrm{G}$ is a Cayley digraph with $\gamma(\mathrm{G}) \geq 2$, then $\gamma(\mathrm{G}) \leq \delta\lceil((\delta(\mathrm{G})) /((\gamma(\mathrm{G})-1))\rceil+1$

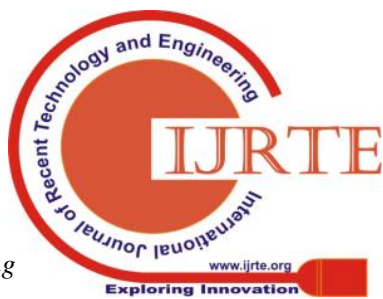




\section{V.SUMMARY AND CONCLUSION}

The Domination of Cayley digraph and compliment of Cayley digraph are discussed and domination of Cayley digraph of Alternating group A4 and its complement are investigated. Through the above study, we found that the domination of complement of Cay $(\mathrm{A} 4, \mathrm{~S})$ with $|\mathrm{S}|=2$ or 3 is always 2 . Then the parameters of the graphs compared and the bound for the Domination number of Cayley digraph is given. In future research, the domination of other Cayley digraphs and its compliment can be found.

\section{REFERENCES}

[1]. J. Lee, Independent perfect domination sets in Cayley graphs, J. Graph Theory 37 (4) (2001) 213-219.

[2]. Italo J. Dejtera and OriolSerrab , Efficient dominating sets in Cayley graphs , Discrete Applied Mathematics , Volume 129 , pp. 319-328, 2003.

[3]. G. S. Domke, J. E. Dunbar, and L. R. Markus, "The inverse domination number of a graph," Ars Combinatoria, vol. 72, pp. 149-160, 2004.

[4]. R.Thamizharasi and R.Rajeswari, "Labelings of Cayley Digraphs and its Line Digraphs", International Journal of Pure and Applied Mathematics, Volume 101 No. 5, 2015, ISSN: 1311- 8080, pp.681-690.

[5]. R.Thamizharasi and R.Rajeswari, "Graceful and Magic Labelings on Cayley Digraphs", International Journal of Mathematical Analysis, Vol. 9, 2015, No. 19, pp. 947-954.

[6]. Parameswari.R, Rajeswari.R, Total magic cordial labeling of $(\mathrm{Pn}, \mathrm{K} 1)$, Gear and Shadow graphs in the International Journal of Pure and Applied Mathematics, Vol.101, 2015, No.6,pp. 993-1001.

[7]. Parameswari.R, Rajeswari.R, Labeling of Quadratic Residue Digraphs over a finite field in Smart Innovation Systems and Technologies, ISSN: 2190-3018, pp.387-396.

[8]. 8. Manjula and R.Rajeswari, "Dominator coloring of Prism graph" in the Journal of Applied Mathematical Sciences(JAMS), Vol. 9, No. 38, 2015, pp. 1889-1894.

[9]. 9. T. Tamizh Chelvam and M. Sivagami, Domination in Cayley graphs: A survey , AKCE International journal of graphs and combinatorics Received 1 May 2017; received in revised form 22 November 2017; accepted 22 November 2017. 